\title{
Evaluation of the Role of Hysteroscopic Endometrial Injury and Its Timing Prior to in vitro Fertilization Treatment
}

\author{
${ }^{1}$ Avani Pillai, ${ }^{2} \mathrm{G}$ Parasuram, ${ }^{3}$ Fessy L Thalakottoor, ${ }^{4} \mathrm{KK}$ Gopinathan
}

\section{ABSTRACT}

Aim: To evaluate the role of hysteroscopic endometrial injury and its timing prior to embryo transfer in patients undergoing IVF (in vitro fertilization) treatment.

Materials and methods: A total of 133 patients who underwent hysteroscopy at CIMAR Fertility Centre at Kochi, between January 2013 and December 2014, and had normal hysteroscopic findings were enrolled for the study. These women subsequently underwent IVF treatment and were evaluated in three groups based on the timing of hysteroscopy before embryo transfer: Group I - hysteroscopy performed 50 days or less before embryo transfer $(n=54)$, group II - hysteroscopy performed between 51 days and 6 months of embryo transfer $(n=45)$, and group III - hysteroscopy performed more than 6 months before embryo transfer $(n=34)$.

Results: The implantation rates were $21.8,22.6$, and $21.6 \%$ in groups I, II, and III respectively. Overall pregnancy rates were $44.4,48.9$, and $44.1 \%$. Clinical pregnancy rates (CPR) were $40.7,46.7$, and $44.1 \%$ and live birth rates (LBR) were 30,29 , and $26 \%$ in groups I, II, and III respectively. Thus the implantation rate, overall pregnancy rate, and CPR were not significantly different in the three groups.

Conclusion: Hysteroscopic endometrial injury prior to IVF does not improve the pregnancy rate in patients with normal hysteroscopic findings.

Clinical significance: Due to the lack of definitive evidence regarding the role of hysteroscopic endometrial injury, ideal technique, and its timing prior to embryo transfer, it is necessary to evaluate the role of endometrial injury as a fertility treatment in women undergoing assisted reproductive technology (ART) cycles, as well as to present it in a way that supports clinical practice. At this stage, there is little evidence to support hysteroscopic endometrial injury prior to embryo transfer as a standard of care, and its use should be limited to selected cases after careful deliberation between the medical team and patient.

\footnotetext{
${ }^{1}$ Assistant Professor and Consultant, ${ }^{2}$ Consultant and Scientific Director, ${ }^{3}$ Infertility Consultant and Laparoscopic Surgeon ${ }^{4} \mathrm{Head}$ and Unit Director

${ }^{1}$ Department of Reproductive Medicine, Amrita Institute of Medical Sciences, Kochi, Kerala, India

${ }^{2,4}$ Department of Reproductive Medicine, CIMAR Fertility Centre Kochi, Kerala, India

${ }^{3}$ Department of Reproductive Medicine, AMMA IVF and Fertility Centre, Kochi, Kerala, India

Corresponding Author: Avani Pillai, Assistant Professor and Consultant, Department of Reproductive Medicine Amrita Institute of Medical Sciences, Kochi, Kerala, India Phone: +919249495565, e-mail: avanimj@gmail.com
}

Keywords: Cohort study, Endometrial injury, Endometrial receptivity, Endometrial scratching, Pre-IVF hysteroscopy.

How to cite this article: Pillai A, Parasuram G, Thalakottoor FL, Gopinathan KK. Evaluation of the Role of Hysteroscopic Endometrial Injury and Its Timing Prior to in vitro Fertilization Treatment. Int J Infertil Fetal Med 2016;7(2):52-57.

\section{Source of support: Nil}

Conflict of interest: None

Date of received: $7-3-2016$

Date of acceptance: 22-5-2016

Date of publication: August 2016

\section{INTRODUCTION}

In vitro fertilization/intracytoplasmic sperm injectionembryo transfer (IVF/ICSI-ET) plays a key role in the treatment of female infertility and male sterility. Despite constant advances in procedures for culturing and transferring embryos over the last two decades, the CPR has remained unchanged (currently only 32.4-33.0\% per IVF transfer as reported by ESHRE [European Society of Human Reproduction and Embryology] in 2010). ${ }^{1}$

Embryo implantation is a complex and multistage process, and inadequate implantation may be one of the causes that may explain outcome failures. ${ }^{2}$ A successful implantation not only depends on quality of embryo but is also related to the endometrial receptivity. Thus, developing a receptive endometrium, which is a limiting step for success in IVF procedure, is at upmost importance for a successful implantation. ${ }^{2,3}$ Of the various methods evaluated to improve endometrial receptivity, local endometrial injury has gained a lot of recent attention.

Although the underlying mechanism of how endometrial injury improves endometrial receptivity remains unknown, many hypotheses have been proposed. One of the hypotheses states that local injury to the endometrium induces endometrial decidualization, which increases the probability of implantation of the transferred embryo. ${ }^{4}$ According to another hypothesis, local injury to the endometrium induces an inflammatory response that provokes a massive secretion of different cytokines and growth factors that play a role in development of a receptive endometrium. ${ }^{5,6}$ Another mechanism states that local endometrial injury in stimulated cycle delays the endometrial development because of the wound repair 
processes, correcting the asynchrony between endometrial and embryo stage. ${ }^{7}$ Endometrial gene modulation post injury is also thought to have a positive effect on adhesion of blastocyst to the endometrium. ${ }^{8}$

Thus, various methods of endometrial injury have been mentioned in the literature, including endometrial biopsy, curettage, and hysteroscopic injury/scratching. Hysteroscopic endometrial injury prior to embryo transfer is a promising procedure and has been suggested as a means to improve the implantation rate in patients undergoing IVF treatment. Whether this improvement in implantation rate has a clinically significant benefit on pregnancy outcome is unknown.

In this study, we have evaluated the role of hysteroscopic endometrial injury and its timing prior to embryo transfer in improving the pregnancy outcome in women undergoing IVF treatment.

\section{AIM}

To evaluate the role of hysteroscopic endometrial injury and its timing prior to embryo transfer in patients undergoing IVF treatment.

\section{MATERIALS AND METHODS}

- Design and duration: Two years retrospective cohort study.

- Study population: A total of 133 women who underwent hysteroscopy at CIMAR Fertility Centre, Kochi, between January 2013 and December 2014 and had normal hysteroscopy findings and subsequently underwent IVF treatment were enrolled for the study.

- Inclusion criteria:

- Women not conceived for at least 3 years.

- Women with no prior term pregnancies.

- Hysteroscopy showing normal findings.

- At least one grade 1 embryo transferred.

- Endometrium of $\geq 7 \mathrm{~mm}$ on the day of human chorionic gonadotropin (hCG).

Demographic characteristics of the couples were recorded. After a basic history and initial clinical assessment, routine transvaginal ultrasound (TVS) with antral follicular count was done. Semen analysis was done for the male partner.

Hysteroscopy was performed in the proliferative phase of the cycle. Rigid hysteroscope (continuous flow, $30^{\circ}$ forward oblique view) assembled in a 4-mm diameter diagnostic sheath with an atraumatic tip (Karl Storz Endoscopy, Germany) was used. An isotonic solution ( $0.9 \%$ normal saline or Ringer's lactate) administrated via a pressure control pump (hysteromat) was used. The pressure was preset at between 80 and $120 \mathrm{~mm} \mathrm{Hg}$ with the aim to use the lowest pressure required to distend the uterine cavity adequately.

Procedure was performed after taking informed consent from the women, and antibiotic prophylaxis was administered as per hospital protocol. Entry into the uterine cavity was under direct vision using gentle manipulation to minimize trauma to the cervical canal with tenaculum/vulsellum applied to the anterior lip of the cervix to help straighten the cervical canal whenever required.

\section{IVF Protocol}

Long protocol was used. Patients were downregulated with a gonadotropin-releasing hormone (GnRH) agonist ( single-dose lupride depot $3.75 \mathrm{mg}$ IM or lupride acetate $0.5 \mathrm{mg}$ daily injections) in the midluteal phase (days 18-21) of the previous cycle. After downregulation was achieved, ovarian stimulation was commenced from cycle day 2 to 3 with gonadotropins (recombinant FSH and/ or human menopausal gonadotropin [hMG]) at a dose of 150 to 300 IU daily according to age, antral follicle count, BMI, and previous cycles, if present for first 7 days. Thereafter, the dose was adapted according to the ovarian response to treatment. The GnRH agonist was continued up to and including the day of administration of hCG. The final oocyte maturation was achieved with 10,000 IU of hCG (Ovunal SC) when two or more follicles reached a diameter of $\geq 17 \mathrm{~mm}$. Oocyte retrieval was performed 36 hours after HCG administration. After oocyte retrieval, ICSI was performed. Embryo transfer was performed on day 3 or 5 after oocyte retrieval.

The luteal phase was supplemented with intramuscular or intravaginal progestrones and, if pregnancy was achieved, continued till 12 weeks of gestation. A quantitative analysis of serum beta-hCG concentrations was conducted 14 days after embryo transfer. A cutoff level of $50 \mathrm{mIU} / \mathrm{mL}$ was used to confirm pregnancy. Subsequently, ultrasound was performed after 6 weeks of gestation to confirm number of sacs implanted and viability. These pregnancies were followed till term.

Women were divided into three groups based on the timing of hysteroscopy from embryo transfer for the purpose of analysis

- Group I: $\leq 50$ days $(\mathrm{n}=54)$

- Group II: 51 days to 6 months $(\mathrm{n}=45)$

- Group III: $>6$ months $(\mathrm{n}=34)$.

Outcome measures were overall pregnancy rate, implantation rate, clinical pregnancy rate (CPR), and live birth rate (LBR), which were compared in the three groups. The pregnancy outcome in the subgroup of women with recurrent implantation failure was also compared in the three groups. 


\section{Definitions}

- Recurrent implantation failure - Failure to achieve a clinical pregnancy after two or more attempts of transfer of good-quality embryos.

- Implantation rate - The number of embryos which have produced ultrasonographic evidence of an intrauterine gestational sac per the total number of embryos transferred into the uterine cavity.

- Overall pregnancy rate - The number of patients with biochemical/clinical pregnancy divided by the number of patients who had embryo transfer.

- Clinical pregnancy rate - The number of patients with clinical pregnancy divided by the number of patients who had embryo transfer. Clinical pregnancy was defined by the ultrasound evidence of fetal heart beat.

- Live birth rate - The number of patients with live births divided by the number of patients who had embryo transfer.

Statistical analysis was done using ANOVA/KruskalWallis test and categorical data was analyzed using chi-square analysis/Fisher's exact test.

\section{OBSERVATION AND RESULTS}

The three study groups were similar in terms of measured baseline characteristics (Table 1).

Comparison of the outcome measures between the three groups of endometrial injury performed $<50$ days, 51 days to 6 months, and $>6$ months prior to embryo transfer (Graph 1).

In our study, the CPR was $40.7,46.7$, and $44.1 \%$ in groups I, II, and III respectively (p-value 0.837 ) and LBR was 29.6, 28.9, and 26.5\% in groups I, II, and III respectively ( $\mathrm{p}$-value 0.949 ). There was no statistically significant difference on comparing the outcome between the individual groups. Thus, the timing of hysteroscopic
Table 1: Baseline characteristics

\begin{tabular}{|c|c|c|c|}
\hline Characteristics & $\begin{array}{l}\text { Group I } \\
(n=54)\end{array}$ & $\begin{array}{l}\text { Group II } \\
(n=45)\end{array}$ & $\begin{array}{l}\text { Group III } \\
(n=34)\end{array}$ \\
\hline Age (years; mean) & 32.39 & 33.18 & 30.79 \\
\hline BMI (mean) & 25.19 & 25.25 & 25.41 \\
\hline $\begin{array}{l}\text { Duration of infertility } \\
\text { (years; mean) }\end{array}$ & 6.4 & 7.5 & 5.2 \\
\hline Male factor infertility (\%) & 43 & 40 & 41 \\
\hline $\begin{array}{l}\text { Recurrent implantation } \\
\text { failure }(\%)\end{array}$ & 29.6 & 40 & 35.3 \\
\hline $\begin{array}{l}\text { Endometrial thickness } \\
(\mathrm{mm} ; \text { mean })\end{array}$ & 9.3 & 8.9 & 9.1 \\
\hline $\begin{array}{l}\text { No. of embryos transferred } \\
\text { (mean) }\end{array}$ & 2.8 & 3.3 & 3 \\
\hline
\end{tabular}

endometrial injury did not show any benefit on pregnancy outcome.

Comparison of the outcome measures between endometrial injury $\leq 6$ months $v s>6$ months prior to embryo transfer (Graph 2).

Since the effect of endometrial injury is unlikely to persist beyond 6 months, we compared the groups based on hysteroscopy done $\leq 6$ months before embryo transfer vs $>6$ months prior to embryo transfer. Implantation rates were $22 \%$ vs $23 \%$ (p-value 0.924 ), CPR $41 \%$ vs $47 \%$ (p-value 0.945 ), and LBR $30 \%$ vs $29 \%$ (p-value 0.753 ) between groups I and II combined and group III. There was no difference found in the pregnancy outcome between the two groups. Hence hysteroscopic endometrial injury in patients without endometrial pathology may not be of benefit in improving pregnancy outcome.

Comparison of the outcome measures between the three groups of endometrial injury performed $<50$ days, 51 days to 6 months, and $>6$ months prior to embryo transfer in women with recurrent implantation failure (Graph 3).

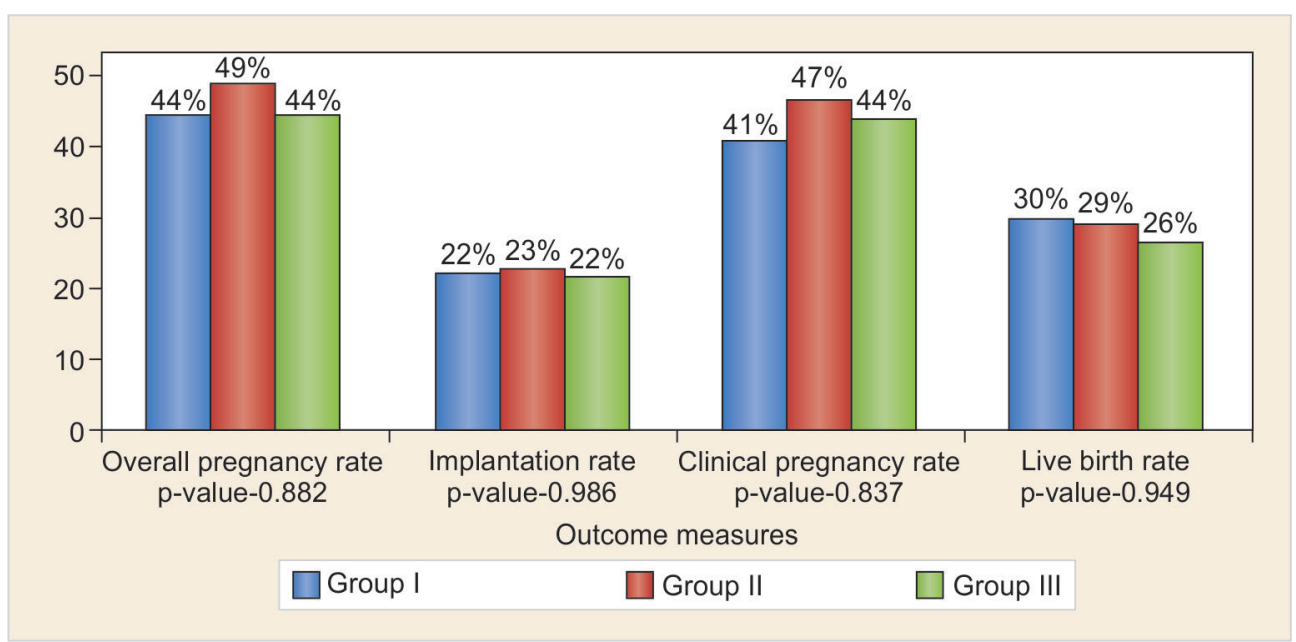

Graph 1: Comparison of the outcome measures between the three groups of endometrial injury performed $<50$ days, between 51 days to 6 months and $>6$ months of embryo transfer 


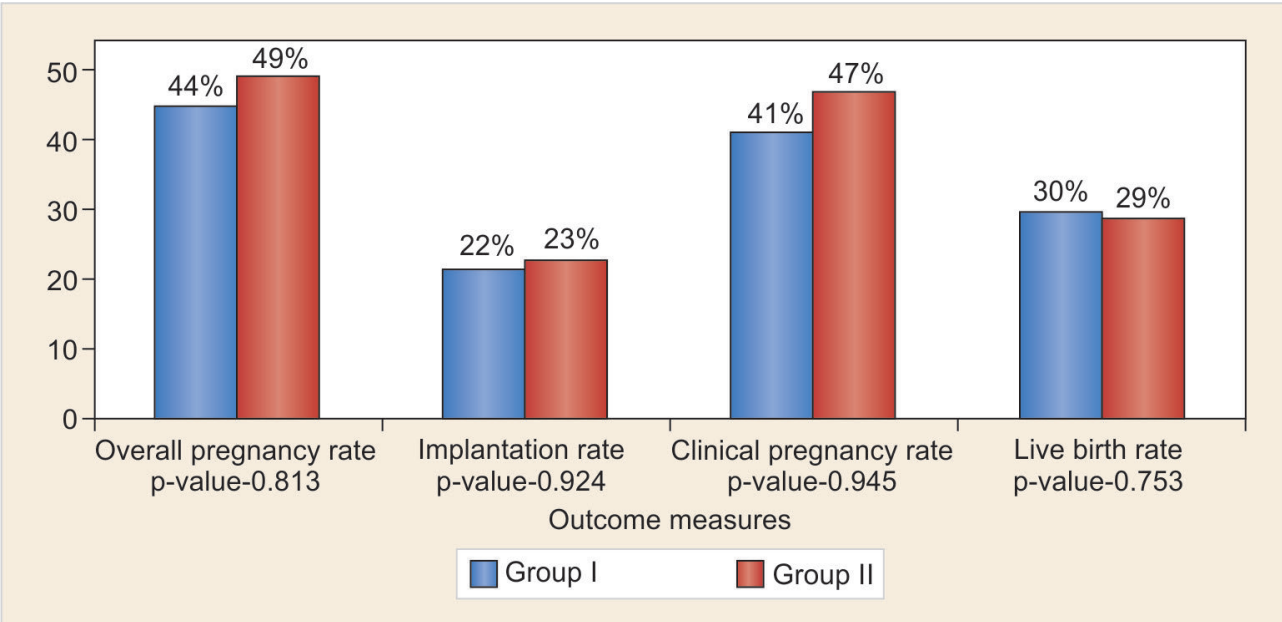

Graph 2: Comparison of the outcome measures between endometrial injury performed $<6$ months $v s>6$ months of embryo transfer

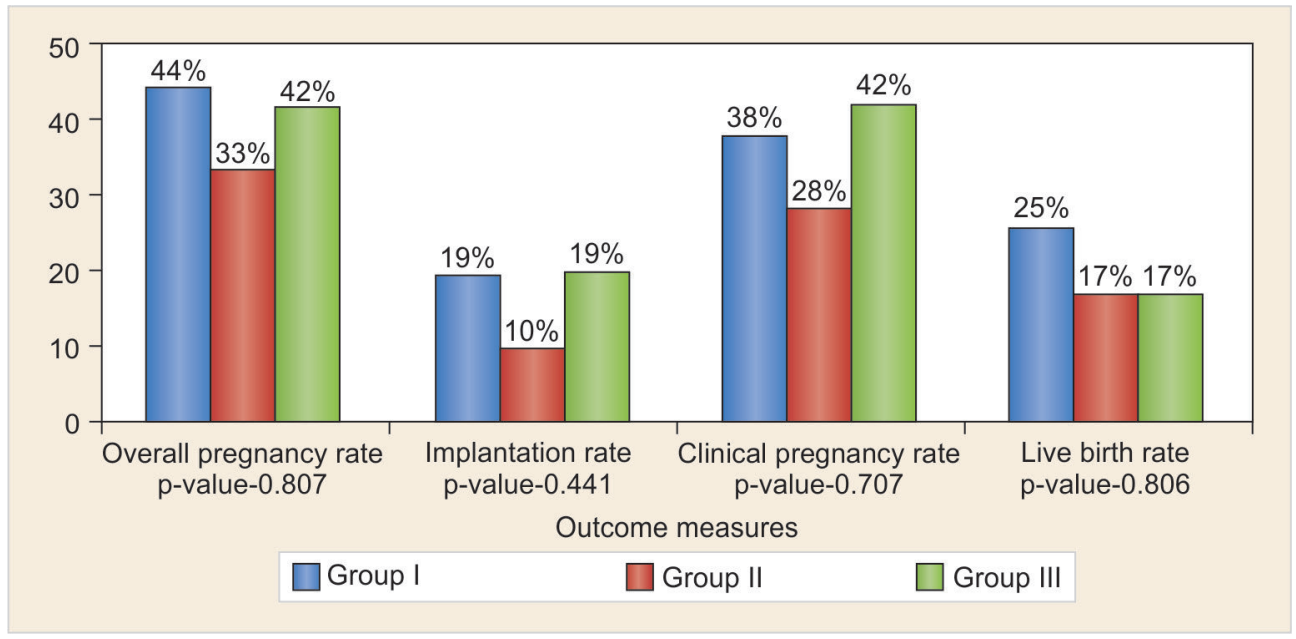

Graph 3: Comparison of the outcome measures between the three groups of endometrial injury performed $<50$ days, between 51 days to 6 months, and $>6$ months of embryo transfer in women with recurrent implantation failure

In the subgroup analysis of women with recurrent implantation failures, CPR were 37.5, 27.8, and $41.7 \%$ (p-value 0.707 ); and LBR were $25,16.7$, and $16.7 \%$ (p-value 0.806). No significant difference in the pregnancy outcome was observed between the three groups of women in which hysteroscopy was done $<50$ days, 51 days to 6 months, and $>6$ months prior to embryo transfer.

Comparison of the outcome measures between endometrial injury $\leq 6$ months $v s>6$ months prior to embryo transfer in women with recurrent implantation failure (Graph 4).

On comparing the effect of hysteroscopic endometrial injury done $\leq 6$ months before embryo transfer vs $>6$ months prior to embryo transfer in patients with recurrent implantation failure, implantation rates were $14.2 \%$ vs $19.4 \%$ (p-value 0.532 ), CPR $32.4 \%$ vs $41.7 \%$ (p-value 0.56 ), and LBR $20.6 \%$ vs $16.7 \%$ (p-value 0.99 ) between groups I and II combined and group III. Results did not show any improvement in the IVF treatment outcome. Thus, hysteroscopic endometrial injury in women with recurrent implantation failure may not be of benefit in improving the pregnancy outcome.

\section{DISCUSSION}

In our study, the overall pregnancy rate, implantation rate, CPR, and LBR were similar in the three groups of immediate, intermediate, and early endometrial injury.

This was comparable to the study conducted by El-Toukhy et al. ${ }^{9}$ The TROPHY Trial reported at the 30th annual meeting of ESHRE in June 2014 was a large randomized trial performed in eight IVF centers in Europe between 2010 and 2013. More than 700 women were randomized to IVF with hysteroscopy (in the preceding cycle), or IVF without; all were under the age of 38, without known uterine pathology, and had history of unsuccessful IVF (two to four failed cycles). 


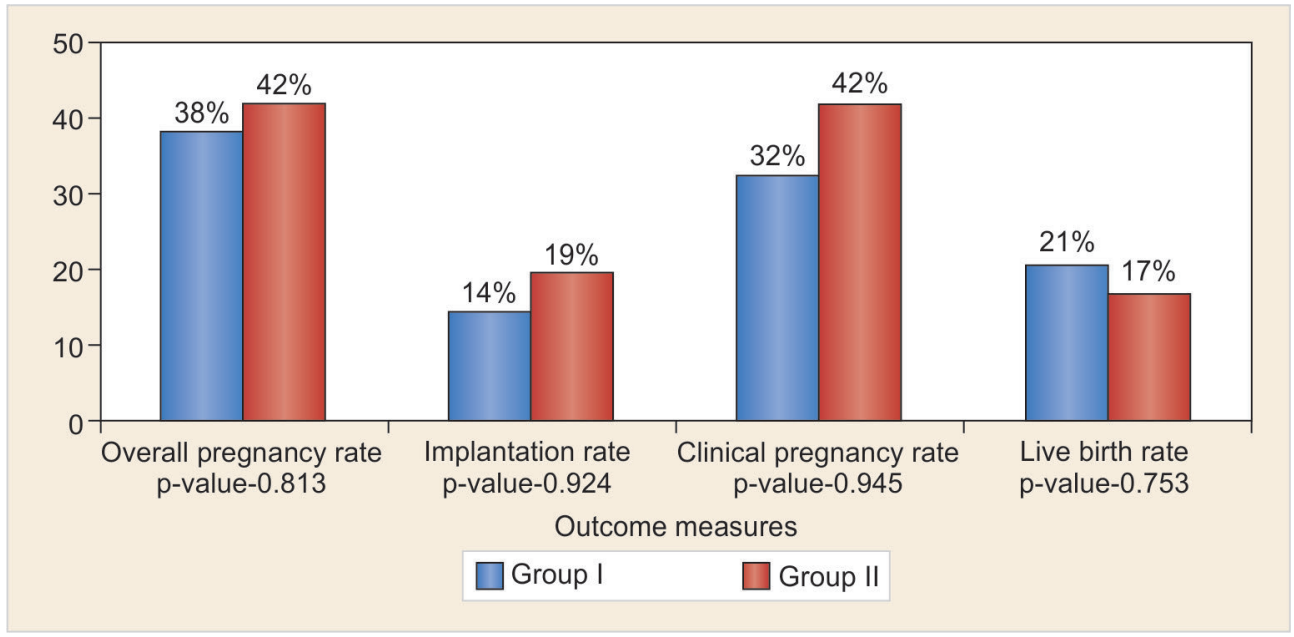

Graph 4: Comparison of the outcome measures between endometrial injury performed $<6$ months vs $>6$ months of embryo transfer in women with recurrent implantation failure

Outcome results following IVF showed no significant difference between the two groups: A LBR per patient of $31 \%$ in the hysteroscopy group and $29 \%$ in the control group. The results were similar to our study and suggest that routine hysteroscopy prior to IVF in women who have experienced two to four failed IVF attempts do not significantly improve the subsequent IVF outcome.

The meta-analysis conducted by Panagiotopoulou et $\mathrm{al}^{10}$ which included four randomized controlled trials (RCTs) found significant clinical heterogeneity with regard to patient characteristics, intervention used, and phase of previous menstrual cycle.

Yeung et $\mathrm{al}^{11}$ conducted an RCT in 2014 including 300 women and found CPR comparable in cases vs control group (CPR 34\% vs 38\%) similar to our study.

Yu et $\mathrm{al}^{12}$ included 215 women who underwent hysteroscopy before starting the first IVF treatment cycle and 284 women who only had TVS prior to starting IVF. The authors reported no significant differences in the CPR ( $43 \%$ vs $44 \%)$, miscarriage rate $(15.2 \%$ vs $16 \%$ ) and LBR ( $34 \%$ vs $35.6 \%$ ) per cycle between the two groups.

However, some data has shown benefits after endometrial injury.

A Cochrane review ${ }^{13}$ in 2015 included 14 paralleldesign RCTs with 1,063 women in the intervention groups and 1,065 women in the control groups. There was moderate-quality evidence to indicate that endometrial injury performed between day 7 of the previous cycle and day 7 of the ET cycle, increases the likelihood of live birth/ ongoing pregnancy and clinical pregnancy (CPR 38.6\% vs $29.8 \%$ and LBR $34.2 \%$ vs $26 \%$ ). Although an overall benefit was observed, subgroup analysis suggested that endometrial injury might benefit only women with two or more previous failures, and 8 of the 14 included studies were deemed to be at high risk of bias in at least one domain. Hence further research is needed to confirm this observation.

A meta-analysis by Pundir et $\mathrm{al}^{14}$ included a total of 3,179 participants. They compared hysteroscopy with no intervention in the cycle preceding the first IVF cycle. There was a significantly higher CPR (relative risk [RR] 1.44, 95\% CI 1.08-1.92, p-value 0.01) and LBR (RR 1.30, 95\% CI 1.00-1.67, p-value 0.05) in the subsequent IVF cycle in the hysteroscopy group. Another observational study by Karayalçin et $\mathrm{al}^{15}$ showed better implantation rates in immediate hysteroscopy group.

Thus, the various studies in the literature evaluating the role of endometrial injury in improving the IVF treatment outcome have significant clinical heterogeneity with regard to patient characteristics and the intervention used. There is conflicting evidence in the literature regarding the definite role of endometrial injury/stimulation as a procedure to improve pregnancy outcome. The consensus regarding the patient selection, the timing of endometrial injury, technique, and number of endometrial stimulation required is lacking. Well-designed RCTs are required to evaluate the role of hysteroscopic endometrial injury prior to embryo transfer in patients with normal prior hysteroscopic findings.

Our study did not show any clinically significant benefit of hysteroscopic endometrial injury prior to embryo transfer in the study population. It has certain limitations including its retrospective design and the small sample size.

\section{CONCLUSION}

- Hysteroscopic endometrial injury prior to embryo transfer in patients with normal hysteroscopy findings may not be beneficial in all women undergoing IVF treatment. 
- The timing of endometrial injury prior to embryo transfer may not have an impact on the pregnancy outcome.

- Hysteroscopic endometrial injury in subgroup of patients with recurrent implantation failure may not be beneficial in improving the pregnancy outcome.

\section{CLINICAL IMPLICATION}

Developing a receptive endometrium is a limiting step for success in IVF procedure and is of utmost importance for a successful implantation. Due to lack of definitive evidence regarding the role of hysteroscopic endometrial injury, ideal technique, and its timing prior to embryo transfer, it is necessary to evaluate the role of endometrial injury as a fertility treatment in women undergoing assisted reproductive technology (ART) cycles, and to present it in a way that supports clinical practice. At this stage, there is little evidence to support hysteroscopic endometrial injury prior to embryo transfer as a standard of care and its use should be limited to selected cases after careful deliberation between the medical team and patient.

\section{REFERENCES}

1. De Mouzon J, Goossens V, Bhattacharya S, Castilla JA, Ferraretti AP, Korsak V, Kupka M, Nygren KG, Nyboe Andersen A. Assisted reproductive technology in Europe, 2006: Results generated from European registers by ESHRE. Hum Reprod 2010 Aug;25(8):1851-1862.

2. Cross JC, Werb Z, Fisher SJ. Implantation and the placenta: Key pieces of the development puzzle. Science 1994 Dec;266(5190):1508-1518.

3. Barash A, Dekel N, Fieldust S, Segal I, Schechtman E, Granot I. Local injury to the endometrium doubles the incidence of successful pregnancies in patients undergoing in vitro fertilization. Fertil Steril 2003 Jun;79(6):1317-1322.

4. Li R, Hao G. Local injury to the endometrium: Its effect on implantation. Curr Opin Obstet Gynecol 2009 Jun;21(3): 236-239.
5. Gnainsky Y, Granot I, Aldo PB, Barash A, Or Y, Schechtman E, Mor G, Dekel N. Local injury of the endometrium induces an inflammatory response that promotes successful implantation. Fertil Steril 2010 Nov:94(6):2030-2036.

6. Gnainsky Y, Granot I, Aldo P, Barash A, Or Y, Mor G, Dekel N. Biopsy-induced inflammatory conditions improve endometrial receptivity. Reproduction 2015 Jan;149(1):75-85.

7. Zhou L, Li R, Wang R, Huang HX, Zhong K. Endometrial injury in COH cycle. Fertil Steril 2008;89(5):1166-1176.

8. Kalma Y, Granot I, Gnainsky Y, Or Y, Czernobilsky B, Dekel N, Barash A. Endometrial biopsy-induced gene modulation: First evidence for the expression of bladder-transmembranal uroplakin $\mathrm{Ib}$ in human endometrium. Fertil Steril 2009 Apr;91(4):1042-1049.

9. El-Toukhy T, Campo R, Sunkara SK, Khalaf Y, Coomarasamy A. A multi-centre randomised controlled study of pre-IVF outpatient hysteroscopy in women with recurrent IVF implantation failure: Trial of Outpatient Hysteroscopy - [TROPHY] in IVF. Reprod Health 2009 Dec;6:20.

10. Panagiotopoulou N, Karavolos S, Choudhary M. Endometrial injury prior to assisted reproductive techniques for recurrent implantation failure: a systematic literature review. Eur J Obstet Gynecol Reprod Biol 2015 Oct;193:27-33.

11. Yeung TW, Chai J, Li RH, Lee VC, Ho PC, Ng EH. The effect of endometrial injury on ongoing pregnancy rate in unselected subfertile women undergoing in vitro fertilization: a randomized controlled trial. Hum Reprod 2014 Nov;29(11): 2474-2481.

12. Yu HT, Wang CJ, Lee CL. The role of diagnostic hysteroscopy before the first in vitro fertilization/intracytoplasmic sperm injection cycle. Arch Gynecol Obstet 2012 Nov;286(5): 1323-1328.

13. Nastri CO, Lensen SF, Gibreel A, Raine-Fenning N, Ferriani RA, Bhattacharya S, Martins WP. Endometrial injury in women undergoing assisted reproductive techniques. Cochrane Database Syst Rev 2015 Mar:(3).

14. Pundir J, Pundir V, Omanwa K, Khalaf Y, El-Toukhy T. Hysteroscopy prior to the first IVF cycle: a systematic review and meta-analysis. Reprod Biomed Online 2014 Feb;28(2): 151-161.

15. Karayalçin R, Ozyer S, Ozcan S, Uzunlar O, Gürlek B, Moraloğlu O, Batioğlu S. Office hysteroscopy improves pregnancy rates following IVF. Reprod Biomed Online 2012 Sep;25(3):261-266. 